\title{
Bilateral Ligation of the Anterior Branch of the Hypogastric Artery in Massive Obstetric Hemorrhage Secondary to Septic Abortion (Case Report)
}

\author{
Angel Millan Juarez ${ }^{1, ~ * ~, ~ C a r l a ~ A m e r i c a ~ S u a r e z ~ J u a r e z ~}{ }^{1, ~ *, ~ A n a ~ E l e n a ~ B a r r i o s ~ H e r a n d e z, ~ " ~}$ \\ Ithamar Milagros Arroyo Martinez ${ }^{3, *}$, Elizabeth Rendon Mondragon ${ }^{4, *}$ \\ ${ }^{1}$ Oncology Gynecology Unit, Military Hospital for Women's Specialties and Neonatology, Mexico City, Mexico \\ ${ }^{2}$ Gynecology Unit, Military Hospital for Women's Specialties and Neonatology, Mexico City, Mexico \\ ${ }^{3}$ Radiology Unit, Military Hospital for Women's Specialties and Neonatology, Mexico City, Mexico \\ ${ }^{4}$ Pathology Unit, Military Hospital for Women's Specialties and Neonatology, Mexico City, Mexico
}

Email address:

millanjuarezangel33@gmail.com (A. M. Juarez),dra.america.suarez@gmail.com (C. A. S. Juarez), anitabarrios_emm@hotmail.com (A. E. B. Hernandez), ithamar_emm@hotmail.com(I. M. A. Martinez), beth_ren@hotmail.com (E. R. Mondragon)

${ }^{*}$ Corresponding author

\section{To cite this article:}

Angel Millan Juarez, Carla America Suarez Juarez, Ana Elena Barrios Herandez, Ithamar Milagros Arroyo Martinez, Elizabeth Rendon Mondragon. Bilateral Ligation of the Anterior Branch of the Hypogastric Artery in Massive Obstetric Hemorrhage Secondary to Septic Abortion (Case Report). Journal of Gynecology and Obstetrics. Vol. 9, No. 3, 2021, pp. 54-58. doi: 10.11648/j.jgo.20210903.11

Received: April 6, 2021; Accepted: April 19, 2021; Published: May 8, 2021

\begin{abstract}
Hemorrhage is the main cause of Maternal Mortality (MM) (27\%) followed by hypertensive disorders and sepsis $(12 \%)$. Septic abortion is considered an intermediate risk factor for the development of Massive Obstetric Hemorrhage (MOH). The algorithm for the management of postpartum hemorrhage due to uterine atony that includes systematic pelvic devascularization has been described, but this management is really planned for resolution of the pregnancy after the 20th week of gestation, since an HMO due to abortion is un usual. We present the case of a 21-year-old patient who self-medicates a prostaglandin analog at 2 months of pregnancy, achieving only a threat of abortion, goes to the emergency room 3 months later with a diagnosis of septic shock, USG and MRI are performed with altered results, only of hepatomegaly, delayed abortion of 8 weeks of evolution and gestational trophoblastic disease. Emergency MVA was performed due to profuse bleeding, placement of a Bakri balloon and clamping of the uterine arteries without results, for which an emergency exploratory laparotomy (LAPE) was performed with ligation of the anterior trunk of the internal iliac artery, being a successful procedure, without the need for Obstetric Hysterectomy (HO). The patient is managed in intensive care and in the end the diagnosis of TSG is ruled out. Bilateral Hypogastric Artery Ligation (BHAL) in the case of Massive Obstetric Hemorrhage (MOH) secondary to delivery or cesarean section is commonly used, however it is not a technique to report when bleeding is secondary to abortion. In these cases, it is also a viable, successful, fertility-preserving surgical procedure, and an alternative to Obstetric Hysterectomy $(\mathrm{OH})$ when other less invasive methods such as uterine artery clamping or Bakri balloon have failed.
\end{abstract}

Keywords: Ligation, Hypogastric, Artery, Massive, Obstetric, Hemorrhage, Incomplete, Abortion, Preserve, Fertility

\section{Introduction}

The World Health Organization (WHO) indicates that approximately 810 women die every day worldwide as a result of complications related to pregnancy or childbirth, and hemorrhage is the leading cause of MM followed by hypertensive disorders and sepsis [1, 2].

A woman dies from $\mathrm{MOH}$ approximately every 4 minutes, which represents approximately $27 \%$ of $\mathrm{MM}$ cases. $\mathrm{MOH}$ is defined as a blood loss $>2 \mathrm{~L}$ and/or a blood volume loss of $30 \%$ to $40 \%$ and/or $>10 \%$ in the hematocrit $(\mathrm{Htc})$ value. It is generally associated with the need for $\mathrm{HO}$, development of Consumption Coagulopathy (CC) and admission to Critical Care 
Units in Obstetrics (CCUO) [2-4].

Septic abortion (SA) is responsible for approximately $12 \%$ of maternal deaths, it is defined as any loss of pregnancy after 20 weeks (spontaneous or induced) complicated by an infection of the upper genital tract. Approximately $8 \%$ to $12 \%$ of obstetric patient admissions to CCUO are due to sepsis. The case fatality rate for maternal sepsis is of $8 \%$ and up to $14 \%$ in septic shock $[4,5]$.

Both pathologies can occur together, SA is considered an intermediate risk factor for the development of an $\mathrm{MOH}$. It is feasible to prevent $\mathrm{MOH}$ in these patients using uterotonic drugs such as oxytocin, however, oxytocin receptors appear from week 13 of pregnancy, so in abortions of 12 weeks or less it is not really useful. The use of ergometrine and carbetocin is ruled out in these patients from 13 to 20 weeks if there is liver failure, and ergometrine is contraindicated in sepsis. [4]

The algorithm for the management of postpartum hemorrhage due to uterine atony includes in the following order: uterine massage, oxytocics, bimanual compression, balloon tamponade, application of modified B-Lynch compression sutures, systematic pelvic devascularization (uterine/ovarian/quadruple/internal iliac), Uterine Artery Embolization (UAE) and finally OH. But this management is really planned for resolution of the pregnancy after the 20th week of gestation, since an $\mathrm{MOH}$ for an abortion is rare [2].

Bakri balloon tamponade is the most common form of surgical treatment in up to $46 \%$ of cases, followed by compression sutures in $24 \%$ and $\mathrm{OH}$ in $4 \%$. Actually, BLABHA is not considered a common technique in pre-term or full-term pregnancies, and less has been described performing it in the case of $\mathrm{MOH}$ due to abortion, hence the importance of presenting this case and giving it as a viable and successful option [6].

\section{Case Report}

A 21-year-old patient with a diagnosis of early pregnancy consumed $800 \mathrm{~g}$ of misoprostol, presented bleeding after 3 days and later presented transvaginal bleeding that she attributed to two normal menstruations. The patient was admitted to the Emergency Department (Emergencies) with sudden abdominal pain in the epigastrium and right upper quadrant, accompanied by intolerance to the oral route, acholia and choluria. Upon examination, blood pressure (BP) of $90 / 56 \mathrm{mmHg}$, jaundice in both scleras and integuments, congestive breasts, hepatomegaly, positive Murphy's sign, vulva and vagina with gestational changes, uterus $8 \times 7 \times 6$ $\mathrm{cm}$, cervix $3 \times 2 \times 2 \mathrm{~cm}$, closed, with pain on mobilization, little transvaginal bleeding, fetid discharge, normal tendon reflexes. Laboratory studies are performed with altered reports of human chorionic gonadotropin (hCG- $\beta$ ) subunit of $56.48 \mathrm{mIU} / \mathrm{ml}$, leukocytes (Leu) of $23 \times 109$ at the expense of neutrophils $80 \%$, hyperbilirubinemia at the expense of direct bilirubin (DB) with a value of $3.5 \mathrm{mg} / \mathrm{dL}$, elevated liver transaminases with Alanine Amino Transferase (ALT) 2262 U / L and Aspartate Amino Transferase (AST) of 1019
$\mathrm{U} / \mathrm{L}$.

Abdominal Ultrasound (US) is requested, which reports a liver with preserved morphology, enlarged in size, homogeneous parenchyma without lesions, without evidence of bile duct dilation; normal gallbladder; both kidneys of normal characteristics (Figure 1). Obstetric USG with suspected invasive mole versus epithelioid trophoblastic tumor due to the presence of endometrium and myometrium of heterogeneous echogenicity with turbulence and increased vascularity, uterine cavity occupied by product with craniocaudal length of $15.4 \mathrm{~mm}$ ( 8 weeks), without heartbeat; ovaries of normal characteristics and volumes (Figure 2). Magnetic Resonance Imaging (MRI) shows a uterus with an intracavitary mass due to probable trophoblast disease with focal loss of interface between myometrium and endometrium, hepatomegaly with $18 \mathrm{~cm}$ right hepatic lobe, congestion and / or hepatic inflammatory process, alithiasic cholescystitis (Figure 3).

During hospital surveillance, the patient was treated with a broad-spectrum antibiotic. On the third day, she presented profuse transvaginal bleeding that led to a state of shock. She was treated urgently with surgery. Manual Vacuum Aspiration (MVA) and Bakri balloon placement were performed, maneuvers with which bleeding persisted. It was decided to perform an EX LAP plus BLPBHA, with adequate control of the hemorrhage.

Description of BLABHA

The sacral promontory is taken as a reference, three to four centimeters lateral to this reference, the retroperitoneum is incised, anatomically the surgeon positions himself on the bifurcation of the common iliac artery. It is worth mentioning that this surgical step results in risk since when prompting we can find the decussation of the ureter and cause an injury. Once the structures have been identified, the retroperitoneum is dissected parallel to the entire course of the hypogastric artery, as well as the surrounding tissue, until its bifurcation is seen in the anterior and posterior trunk, the peripheral tissue of the anterior trunk. It is safely dissected until it is free and it is ligation with 1-0 catgut absorbable suture. Haemostasis is verified and the same contralateral procedure is performed (Figure 4) [7, 8].

\section{Results}

A $2500 \mathrm{ml}$ bleeding was quantified, 5 erythrocyte concentrates, and 3 packages of fresh plasma were transfused since the patient was undergoing Disseminated Vascular Coagulopathy (CVD) and the postoperative period continued in the intensive care unit.

The result of the pathology describes trophoblastic remains with chorionic villi from the first trimester of gestation with moderate edema and hair necrosis, presence of trophoblast, membranes and decidua with acute and chronic intense chorioamnioitis, no histopathological characteristics of gestational trophoblastic disease are identified. (Figure 5)

During his surveillance, laboratories are requested with $\mathrm{Hb}$ of $11 \mathrm{~g} / \mathrm{dL}$, Leu 8.7 x 109, Neu 60\%, BD $0.5 \mathrm{mg} / \mathrm{dL}$, ALT 


\section{$147 \mathrm{U} / \mathrm{L}$ and AST $39 \mathrm{U} / \mathrm{L}$.}

The patient was discharged 5 days after admission to the CCUO, and a subdermal implant is placed as a family planning method as the patient is in stable condition.

\section{Discussion}

SA is a life-threatening infection associated with unsafe abortion practices.

The physiological, immunological and mechanical changes that occur during pregnancy make women more susceptible to infections and the signs and symptoms of sepsis are mimicked, causing a delay in the recognition and treatment of sepsis [9, 10].

This patient explicitly presented with a motley pattern of sepsis secondary to retention of products of conception after misoprostol induction of abortion without careful follow-up. The age of the patient and the experience of the physicians in obtaining a quick and accurate diagnosis were favorable variables during her study and prognosis; however, the risk of $\mathrm{MOH}$ was present during hospital stay until the patient was confined to hypovolemic shock. In the emergency situation and given the risk of other complications (CVD and death), we decided to perform EX LAP and BLABHA as previously described, thus achieving containment of the hemorrhage and better still, preservation of fertility.

The Bakri balloon has a success rate of $87.5 \%$, however this is in pre-term or full-term pregnancy, in the case of our patient with a delayed abortion of 8 weeks, it was not useful [2].

BLHA's requirement rate is estimated at 62 out of 10,000 births. The mortality rate among patients referred from a first or second level of care is $10.25 \%$, while this rate is $1.11 \%$ among patients who were initially treated at a third level of care. In our case, the patient came from the beginning to our 3rd level institution [11].

BLHA in patients with uterine atony, placental abruption, vaginal/ cervical laceration, uterine rupture, and placenta accreta is effective on its own in $88.9 \%$ of cases. Conservative treatment of postpartum hemorrhage secondary to placenta previa-accreta with BLHA and endouterine hemostatic sutures in the lower uterine segment prevents $\mathrm{OH}$ in up to $84.3 \%$ of patients. We did not find in the literature a percentage of effectiveness of the technique in $\mathrm{MOH}$ due to abortion $[12,13]$.

The first to perform such a ligation was Kelly in 1894, the procedure is not widely used today; stated that BLHA completely cuts off the entire pelvic circulation and this is still the general belief. However, this is not true. There are three specific pairs of collateral arteries, these are: iliolumbar, lateral sacral, and middle hemorrhoidal. This technique is performed because it is known that only proximal ligation ( $2 \mathrm{~cm}$ from the common iliac bifurcation) decreases blood flow by up to $50 \%$ distal to the ligation; Because the collateral circulation provided by the posterior trunk of the internal iliac artery reperfuses the anterior trunk, the ischemic goal is not fully achieved. To date, we have performed 31 procedures, 18 in proximal ligation with the posterior trunk, and thirteen with exclusively anterior trunk ligation, all for obstetric, gynecological and oncological, prophylactic and/or therapeutic indications; we have only observed 2 complications, and they have been resolved, consisting of laceration of the external iliac vein and lower limb paresthesias, without further complications [14, 15].

Patients who end up in $\mathrm{OH}$ due to percrete from the placenta, but if BLHA is performed, they have less blood loss and therefore require less transfusion of units of blood products, so it is also useful. [15]

UAE can be considered in the treatment of hemodynamically stable, unstable patients and even in patients with CVD. The fertility rate after this procedure is good at $60.9 \%$ with a live birth rate of $43.5 \%$. The percentage of fertility after BLHA is also not described in the studies $[16,17]$.

If all attempts to stop the bleeding have failed, HO should be performed as a last resort and life-saving measure. The decision to perform a hysterectomy, while devastating for patients, should not be delayed in cases of hemodynamic instability [2].

$\mathrm{MOH}$ is a public health problem, for which the OB / GYN doctor must be prepared to prevent it by identifying risk factors from their first care, and must be trained to perform unusual techniques such as BLHA, which can mean life and the obstetric future of the patient, even being able to perform abortion from $\mathrm{MOH}$.

\section{Conclusions}

BLABHA in case of $\mathrm{MOH}$ secondary to childbirth or cesarean section is commonly used, however, it is not a technique that reports when bleeding is secondary to abortion. In these cases, it is also a viable, successful, fertility-preserving and alterative surgical procedure to $\mathrm{HO}$ when other less invasive methods such as uterine artery clamping or the Bakri balloon have failed.

\section{Abbreviations}

Bilateral Ligation of the Anterior Branch of the Hypogastric Artery (BLABHA)

Bilateral Ligation of the Hypogastric Artery (BLHA)

Massive Obstetric Hemorrhage (MOH)

Maternal Mortality (MM)

Uterine Artery Embolization (UAE)

Exploratory Laparotomy (EX LAP)

Obstetric Hysterectomy $(\mathrm{OH})$

Critical Care Unit in Obstetrics (CCUO)

Hemoglobin $(\mathrm{Hb})$

Hematocrit (Htc)

Consumption Coagulopathy (CC)

Blood Pressure (BP)

Emergency Room (ER)

Human chorionic gonadotropin $\beta$-subunit (hCG- $\beta$ )

Blood Pregnancy Test (BPT) 
Leukocytes (Leu)

Direct Bilirubin (DB)

Alanine Amino Transferase (ALT)

Aspartate Amino Transferase (AST)

Ultrasound (US)

Manual Vacuum Aspiration (MVA)

Disseminated Vascular Coagulopathy (CVD)

\section{Conflict of Interests}

All authors have no potential conflict of interest.

\section{Financing}

The authors had no funding for this article.

\section{Appendix}

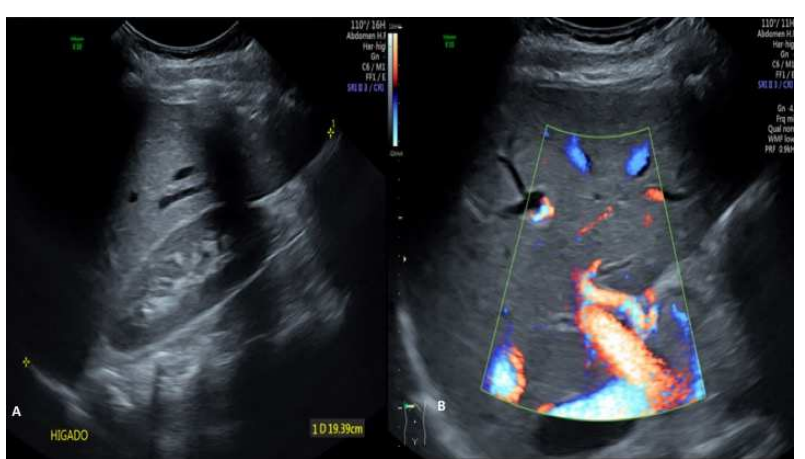

Figure 1. Liver ultrasound: A) Image showing hepatic medial-lateral diameter of $19 \mathrm{~cm}$, in relation to hepatomegaly; liver echogenicity is homogeneous, preserved, without focal or diffuse lesions inside. B) Color Doppler application, where adequate vascularity of the hepatic vessels is shown; no focal or diffuse lesions inside; the intrahepatic bile structures of conserved caliber and morphology.

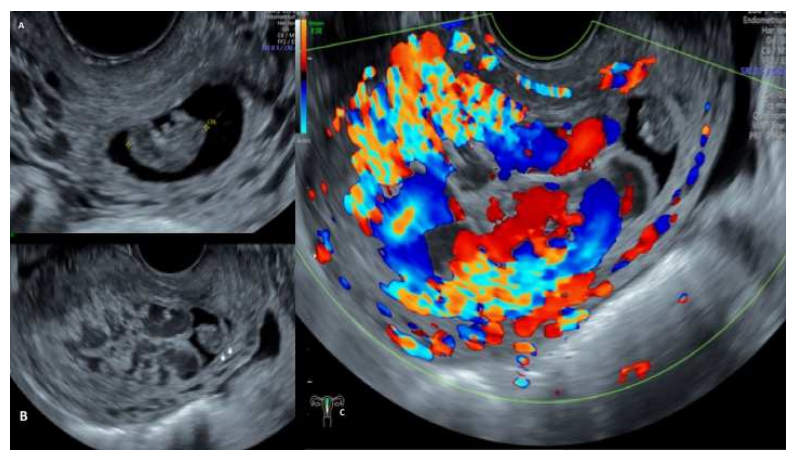

Figure 2. A) Transvaginal ultrasound showing intrauterine pregnancy with cephalocaudal diameter of $15.4 \mathrm{~mm}$, compatible with 8 weeks of gestation, with normal morphology and characteristics, gestational sac and amniotic fluid without alterations. B) Transvaginal ultrasound showing intrauterine pregnancy, located at the level of the uterine body; embryo and gestational sac with normal characteristics; Heterogeneous, thickened placental implantation site occupies the entire uterine fundus and shows loss of the interface with the myometrium. C) Transvaginal ultrasound shown in B), with color Doppler application that shows an important placental venous and diffuse arterial vascular signal, with a predominance at the myometrial interface site.

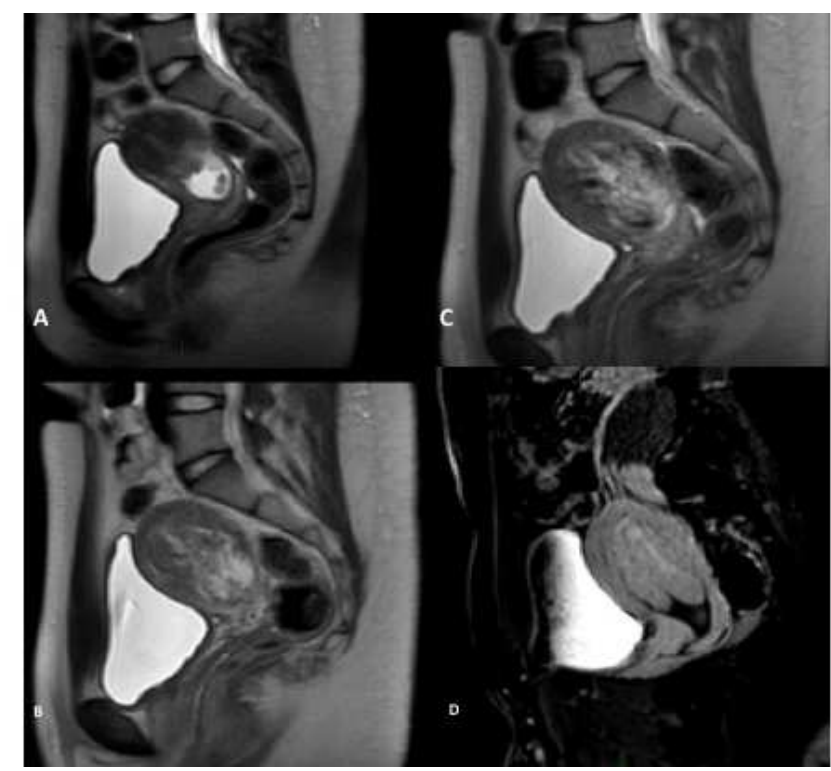

Figure 3. MRI of the pelvis where it is observed: $A, B, C)$ Sagittal T2: Intrauterine pregnancy located at the level of the body region; embryo and gestational sac with normal characteristics; Heterogeneous placental implantation site, thickened, occupies the entire uterine fundus with multiple vascular images in its interior; shows loss of interface with the myometrium. D) Sagittal T2 fat sat: Intrauterine pregnancy located at the level of the body region; embryo and gestational sac with normal characteristics; Heterogeneous placental implantation site, thickened, occupies the entire uterine fundus, shows the interface with the myometrium.

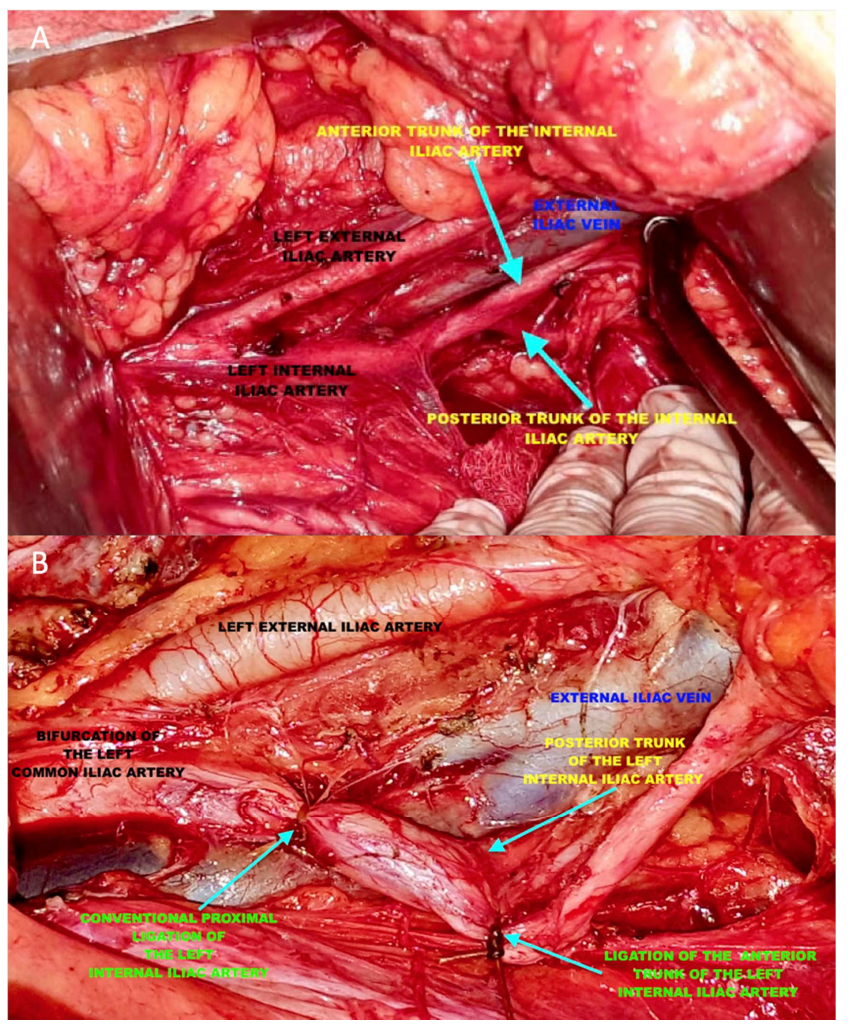

Figure 4. Ligation technique of the anterior trunk of the Internal Iliac Artery. A) Dissection of the pelvic structures. B) Ligation of the anterior trunk. 


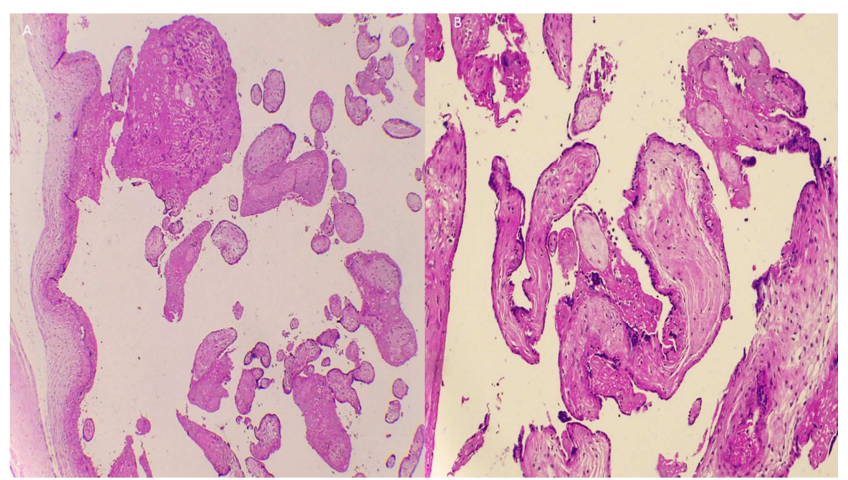

Figure 5. Definitive histopathological report. A) Amnion, Decidua with necrosis and $B$ ) Chorionic villi of the first trimester.

\section{References}

[1] World Health Organization (WHO). Maternal mortality. http://www.who.int/mediacentre/factsheets/fs348/en/.

[2] Sebghati M, Chandraharan E. An update on the risk factors for and management of obstetric haemorrhage. Womens Health $\begin{array}{lllll}\text { (Lond). } 2017 \quad \text { Aug; } 13 & \text { (2): } \quad 34-40 .\end{array}$ http://doi.org/10.117/1745505717716860.

[3] Guasch E, Gilsanz F. Massive obstetric hemorrhage: Current approach to management. Med Intensiva. 2016 Jun-Jul; 40 (5): 298-310. English, Spanish. http://doi.org/10.1016/j.medin.2016.02.010.

[4] O'Brien KL, Shainker SA, Lockhart EL. Transfusion Management of Obstetric Hemorrhage. Transfus Med Rev. $2018 \quad$ Oct; $32 \quad$ (4): $249-255$ http://doi.org.10.1016/j.tmrv.2018.05.003.

[5] Udoh A, Effa EE, Oduwole O, Okusanya BO, Okafo O. Antibiotics for treating septic abortion. Cochrane Database Syst Rev. 2016 Jul 1; 7 (7): CD011528. http://doi.org/10.1002/14651858.CD011528.pub2.

[6] Fadel MG, Das S, Nesbitt A, Killicoat K, Gafson I, Lodhi W, Yoong W. Maternal outcomes following massive obstetric haemorrhage in an inner-city maternity unit. J Obstet Gynaecol. 2019 Jul; 39 (5): 601-605. http://doi.org/10.1080/01443615.2018.1534814.

[7] Abha Sungh, Ruchi Kishore, Saveri Sarbhai Saxena. Ligating internal iliac artery: succes beyond hesitation. The Journal of Obstetrics and Gynecology of India, 2016.
[8] Baggish M. Karram M. Atlas of Pelvic Anatomy and Gynecologic surgery, 4th edition, Elsevier; 2016.

[9] Sherpa D, Johnson BD, Ben-Youssef L, Nagdev A. Diagnosis of Septic Abortion with Point-of-care Ultrasound. Clin Pract Cases Emerg Med. 2017 Jul 6; 1 (3): 268-269. http://doi.org/10.5811/cpcem.2017.3.33574.

[10] Escobar MF, Echavarría MP, Zambrano MA, Ramos I, Kusanovic JP. Maternal sepsis. Am J Obstet Gynecol MFM. $2020 \quad$ Aug; $\quad 2 \quad 100149$. http://doi.org/10.1016/j.ajogmf.2020.100149.

[11] İçen MS, Findik FM, Akin Evsen G, Ağaçayak E, Yaman Tunç S, Evsen MS, Gül T. Hypogastric artery ligation in postpartum haemorrhage: a ten-year experience at a tertiary care centre. J Obstet Gynaecol. 2020 Jun 4: 1-5. http://doi.org/10.1080/01443615.2020.1755623.

[12] Wang CY, Pan HH, Chang CC, Lin CK. Outcomes of hypogastric artery ligation and transcatheter uterine artery embolization in women with postpartum hemorrhage. Taiwan J Obstet Gynecol. 2019 Jan; 58 (1): 72-76. http://doi.org/10.1016/j.tjog.2018.11.014.

[13] Rauf M, Ebru C, Sevil E, Selim B. Conservative management of post-partum hemorrhage secondary to placenta previaaccreta with hypogastric artery ligation and endo-uterine hemostatic suture. J Obstet Gynaecol Res. 2017 Feb; 43 (2): 265-271. http://doi.org/10.1111/jog.13215.

[14] Millán Juárez Á, et al. Ligadura de arterias hipogástricas proximales y tronco posterior bilaterales como profilaxis en histerectomía. Clin Invest Gin Obst. 2020. https://doi.org/10.1016/j.gine.2020.07.002.

[15] Kuhn T, Martimucci K, Al-Khan A, Bilinski R, Zamudio S, Alvarez-Perez J. Prophylactic Hypogastric Artery Ligation during Placenta Percreta Surgery: A Retrospective Cohort Study. AJP Rep. 2018 Apr; 8 (2): e142-e145. http://doi.org/10.1055/s-0038-1666793.

[16] Dinc G, Oğuz Ş. The efficacy of pelvic arterial embolisation for the treatment in massive vaginal haemorrhage in obstetric and gynaecological emergencies: a single-centre experience. J Obstet Gynaecol. 2019 Aug; 39 (6): 774-781. http://doi.org/10.1080/01443615.2019.1586858.

[17] Toguchi M, Iraha Y, Ito J, Makino W, Azama K, Heianna J, Ganaha F, Aoki Y, Murayama S. Uterine artery embolization for postpartum and postabortion hemorrhage: a retrospective analysis of complications, subsequent fertility and pregnancy outcomes. Jpn J Radiol. 2020 Mar; 38 (3): 240-247. http://doi.org.10.1007/s11604-019-00907-2. 